\title{
A meta-analysis of pemetrexed-based doublet compared with pemetrexed alone for the second-line treatment of advanced non-small-cell lung cancer
}

\author{
Sun $\mathrm{CT}^{1}$, Xu X', Sheng $\mathrm{W}^{1}$, Wang $\mathrm{XW}^{1}$, Wen $\mathrm{SL}^{2}$, Han $\mathrm{JQ}^{1}$ \\ ${ }^{1}$ Department of Tumor Research and Therapy Center, Provincial Hospital affiliated to Shandong University, \\ Shandong University, China. hanjq1960@126.com
}

\begin{abstract}
Purpose: This meta-analysis investigated pemetrexed-based doublet compared with pemetrexed alone as second-line therapy for patients with advanced non-small cell lung cancer.

Methods: Randomized controlled trials which compared pemetrexed-based doublet with single-agent pemetrexed in patients as second-line treatment of advanced non-small cell lung cancer were searched. Overall survival (OS) was the primary end point, while secondary end points included progression-free survival, overall response rate, 1 -year survival rate, and grade 3 or 4 toxicity.

Results: Four eligible randomized clinical trials including 1,084 patients were selected. Meta-analysis demonstrated that pemetrexed-based doublet arm significantly improved the overall response rate $(\mathrm{OR}=2.70,95 \% \mathrm{Cl}$ : 1.76-4.15, $p=0.000$ ), compared with docetaxel alone group, while there were no significant differences in overall survival ( $\mathrm{HR}=0.88,95 \% \mathrm{Cl}: 0.74-1.04, \mathrm{p}=0.132)$, progression-free survival $(\mathrm{HR}=0.91,95 \% \mathrm{Cl}: 0.73-1.15$, $\mathrm{p}=0.443)$, and 1 -year survival rate $(\mathrm{OR}=1.43,95 \% \mathrm{Cl}: 0.85-2.40, \mathrm{p}=0.178)$ between the two arms. However, there were more frequencies of grade $3-4$ leucopenia $(\mathrm{OR}=2.86,95 \% \mathrm{Cl}: 1.32-6.20, \mathrm{p}=0.008)$, neutropenia $(\mathrm{OR}=2.69,95 \% \mathrm{Cl}: 1.55-4.68, \mathrm{p}=0.000)$ and thrombocytopenia $(\mathrm{OR}=6.92,95 \% \mathrm{Cl}: 2.51-19.07, \mathrm{p}=0.000)$ in pemetrexed-based doublet group. Grade $3-4$ anemia $(\mathrm{OR}=0.62,95 \% \mathrm{Cl}: 0.33-1.18, \mathrm{p}=0.144)$ and fatigue $(\mathrm{OR}=1.15,95 \% \mathrm{Cl}: 0.73-1.79, \mathrm{p}=0.550)$ had equivalent incidences in the two groups.

Conclusions: This is the first meta-analysis to compare pemetrexed-based doublet with single-agent pemetrexed in second-line therapy of non-small cell lung cancer. Our meta-analysis suggested that pemetrexed combination chemotherapy was not superior to single-agent arm and was not recommended as the second-line chemotherapy for patients with non-small cell lung cancer (Tab. 2, Fig. 6, Ref. 20). Text in PDF www.elis.sk.

Key words: non-small-cell lung cancer, docetaxel, second-line therapy, meta-analysis.
\end{abstract}

Lung cancer is one of the most common malignancies worldwide both in incidence and mortality, and leads in causing cancerrelated deaths throughout the world $(1,2)$. Non-small-cell lung cancer (NSCLC), including squamous cell carcinoma, adenocarcinoma, and large-cell carcinoma accounts for approximately 80 $\%$ of all lung tumors, while $65-80 \%$ of them present as local advanced or metastatic disease $(3,4)$. Less than $5 \%$ of the five-year survival rate is detected in stages IIIB and IV NSCLC patients (5). The treatment for local advanced or metastatic lung cancer is limited. Since surgical excision is impossible in most patients, chemotherapy remains the mainstay of treatment (2).

For recurrent NSCLC patients, second-line chemotherapy is necessary. Docetaxel alone is the current standard for second-line treatment of advanced NSCLC, which can prolong survival after platinum-based chemotherapy $(6,7)$. However, in a randomized

${ }^{1}$ Department of Tumor Research and Therapy Center, Provincial Hospital affiliated to Shandong University, Shandong University, China, and ${ }^{2}$ Department of Health Care, Shandong Province Qianfoshan Hospital Affiliated to Shandong University, Shandong University, China

Address for correspondence: J.Q. Han, 324 Jingwu Weiqi Road, Jinan, Shandong 250021, P. R. China.

Phone: +86.531 .68776100$ phase III trial, Nasser Hanna et al (8) compared the efficacy and toxicity of pemetrexed versus docetaxel in advanced NSCLC patients previously treated with chemotherapy, and concluded that patients treated with pemetrexed have equivalent efficacy outcomes and fewer side effects compared with those obtained with docetaxel. Barlési $\mathrm{F}$ et al (9) also confirmed that docetaxel and pemetrexed shared comparable efficacy, however with a presumably better safety profile in case of pemetrexed. Accordingly, pemetrexed has been approved to be a standard treatment option of second-line therapy in NSCLC (10). Either docetaxel monotherapy or pemetrexed alone has several limitations with median survival times of approximately 8 months and 1-year survival of $30 \%$ (11).

Combination chemotherapy may be one of the considerable measures possibly combining several different kinds of agents and enhancing the anti-tumor effect. On the basis of this strategy, several randomized trials have been conducted to compare pemetrexed-based doublet with pemetrexed alone chemotherapy for the second-line treatment of advanced non-small-cell lung cancer $(11,12)$. However, the conclusions of some studies are contradictory, the sample size in each single trial is small, and the statistical power is inadequate. The latter drawbacks will conceal the potentially relevant differences in efficacy. 
Tab. 1. Baseline characteristics of the four included studies.

\begin{tabular}{|c|c|c|c|c|c|c|c|}
\hline First author & Year & Design & Jadad scores & $\mathrm{n}$ & Median age & Stage IV $(\%)$ & Group \\
\hline \multirow[t]{2}{*}{ Smit EF [11] } & 2009 & Phase II & 3 & 119 & 59 & 77 & $\begin{array}{l}\text { Pemetrexed } 500 \mathrm{mg} / \mathrm{m} 2 \mathrm{iv}, \mathrm{q} 3 \mathrm{w} \text { plus carboplatin area under } \\
\text { the curve } 5 \mathrm{iv} \text {, on day } 1, \mathrm{q} 3 \mathrm{w}\end{array}$ \\
\hline & & & & 121 & 59 & 77 & Pemetrexed alone $500 \mathrm{mg} / \mathrm{m} 2$ iv, $\mathrm{q} 3 \mathrm{w}$ \\
\hline \multirow[t]{2}{*}{ Chiappori A [14] } & 2010 & Phase II & 4 & 80 & 62.1 & 76.3 & $\begin{array}{l}\text { Pemetrexed } 500 \mathrm{mg} / \mathrm{m} 2 \text { iv, on day } 1 \text { of } 21 \text {-day cycles (day } \\
8 \text { in cycle 1) plus oral enzastaurin } 250 \mathrm{mg} \text { bid }\end{array}$ \\
\hline & & & & 80 & 60.7 & 67.5 & $\begin{array}{l}\text { Pemetrexed } 500 \mathrm{mg} / \mathrm{m} 2 \text { iv, on day } 1 \text { of } 21 \text {-day cycles (day } \\
8 \text { in cycle 1) plus placebo }\end{array}$ \\
\hline \multirow[t]{2}{*}{ de Boer RH [15] } & 2011 & Phase III & 5 & 256 & 60 & 86 & $\begin{array}{l}\text { Pemetrexed } 500 \mathrm{mg} / \mathrm{m} 2 \text { iv, } \mathrm{q} 3 \mathrm{w} \text { plus oral vandetanib } 100 \\
\mathrm{mg} \text { qd }\end{array}$ \\
\hline & & & & 278 & 60 & 83 & Pemetrexed $500 \mathrm{mg} / \mathrm{m} 2$ iv, q3w plus oral placebo \\
\hline \multirow[t]{2}{*}{ Schiller JH [12] } & 2010 & Phase II & 3 & 100 & no & 84.5 & $\begin{array}{l}\text { Pemetrexed } 500 \mathrm{mg} / \mathrm{m} 2 \text { iv, on day } 1, \mathrm{q} 3 \mathrm{w} \text { plus matuzumab } \\
800 \mathrm{mg} / \mathrm{m} 2 \text { iv, gw or } 1600 \mathrm{mg} / \mathrm{m} 2 \text { iv } \mathrm{g} 3 \mathrm{w}\end{array}$ \\
\hline & & & & 50 & no & 88 & Pemetrexed alone $500 \mathrm{mg} / \mathrm{m} 2$ iv, on day $1, \mathrm{q} 3 \mathrm{w}$ \\
\hline
\end{tabular}

The aim of this meta-analysis including 4 randomized controlled trials is to compare the efficacy and adverse events of pemetrexed-based doublet with single-agent pemetrexed, and to assess whether pemetrexed-based doublet has an increased efficacy as second-line therapy in the treatment of patients with advanced NSCLC.

\section{Methods}

\section{Literature search}

To ensure the retrieval of all possible trials, we searched the electronic database of PubMed (up to February 2012) and Embase (1980-February 2012) and the Cochrane Register of Controlled Trials as well as abstracts from the conference proceedings of the American Society of Clinical Oncology (ASCO) and European Society of Medical Oncology (ESMO) in recent 10 years. The following different combinations of keywords were used for the search: "NSCLC", "pemetrexed", "second-line therapy", "randomized". The published languages were not limited. The citation list from relevant review articles and selected studies were also manually searched.

\section{Selection criteria}

In order to screen the relevant clinical trials, the following inclusion criteria were performed: (a) patients with pathological confirmation of NSCLC in clinical III-IV stage and previously treated; (b) clinical trials comparing pemetrexed alone with pemetrexed-based doublet chemotherapy; (c) phase II and III randomized controlled trials (RCT); (d) the study with sufficient data for extraction of OS, progression-free survival (PFS), overall response rate (ORR), 1-year survival rate and grade 3 or 4 toxicity. The exclusion criteria were trials that were ongoing studies, interim analyses, brief communication and non-randomized studies such as retrospective studies, letters to the editor, and reviews.

\section{Quality assessment}

Jadad composite scale reported by Moher D et al (13) was the standard to assess the quality of the eligible studies in the meta analysis, which was a five-point scale according to following three questions as to: (a) whether an appropriate randomization method was reported (0-2 scores); (b) whether an appropriate blinding method was reported ( $0-2$ scores); (c) whether withdrawals and dropouts were reported ( $0-1$ scores).

\section{Data extraction}

The following data were extracted from each of the eligible studies: basic information such as first author's name, publication year, journal name, characteristics of patients (sex, age and study duration), follow-up period, inclusion and exclusion criteria, study design, sample size per arm, number of cases and eligibility for evaluation, chemotherapy regimens, doses and schedules of chemotherapy, withdrawals, OS, PFS, ORR, 1-year survival rate and adverse events (AEs). All above information was extracted independently by two investigators and verified in accordance with the inclusion criteria. The primary end point was the overall survival (OS). Secondary end points included progression-free survival (PFS), overall response rate (ORR), 1-year survival rate, and grade 3 or 4 toxicity.

\section{Data analysis and statistical methods}

The Hazard ratio (HR) was used to estimate the overall survival (OS) and progression-free survival (PFS). And the odds ratio (OR) was used for overall response rate, 1-year survival rate, and grade 3 or 4 toxicity. Data analyses were conducted with Stata version 11.0 software (Stata Corporation, College Station, TX, USA) and

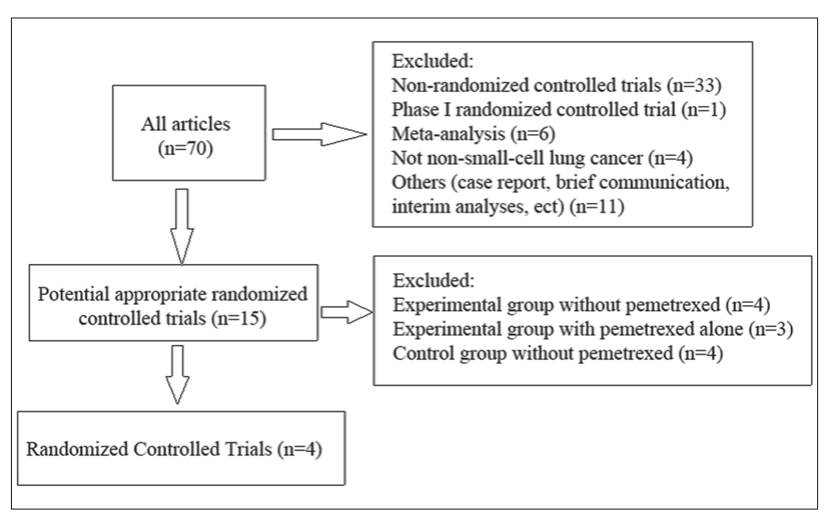

Fig. 1. Flow chart of eligible studies. 


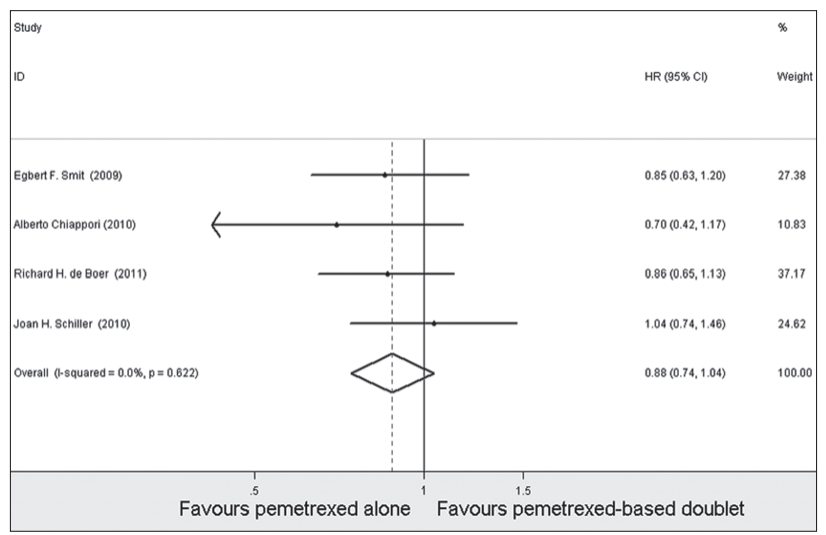

Fig. 2. Forest plot from meta-analysis of overall survival associated with pemetrexed-based doublet compared with pemetrexed alone.

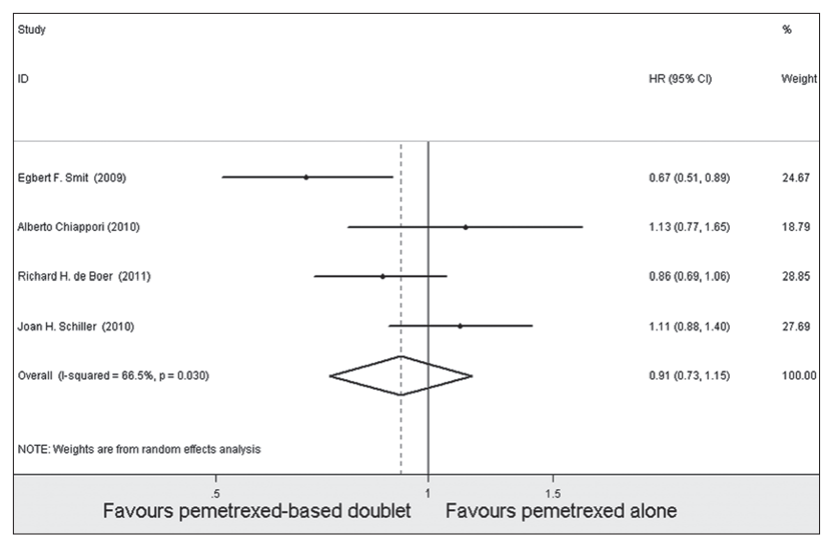

Fig. 3. Forest plot from meta-analysis of progression-free survival associated with pemetrexed-based doublet compared with pemetrexed alone.

values of $p<0.05$ were considered statistically significant. $H R>1$ reflects more deaths or progression in the pemetrexed-based doublet chemotherapy schedule while OR $>1$ reflects more toxicities, 1-year survival rate, and overall response rate in pemetrexed-based doublet arm. The heterogeneity was calculated using the chi-square based Q-test ( $\mathrm{p}<0.05$ or $\mathrm{I}^{2}>50 \%$ was considered statistically significant). Sensitivity analyses were performed to test the possible reasons of heterogeneity in several studies such as study quality, study design and ethnic composition. When heterogeneity was considered statistically significant, a random-effects model was used to analyze the data in meta-analysis. While heterogeneity was absent, a fixed-effects model was used. Begg and Egger tests were used to evaluate publication bias, in which a funnel plot could show whether there was a bias. Statistical tests for heterogeneity, effect estimates, and publication bias were two-sided.

\section{Results}

\section{Study characteristics}

The flow chart is showed in Figure 1. Finally, four trails were eligible for meta-analysis $(11,12,14,15)$, while one was Phase III trial and three were phase II trials. There were 1,084 patients

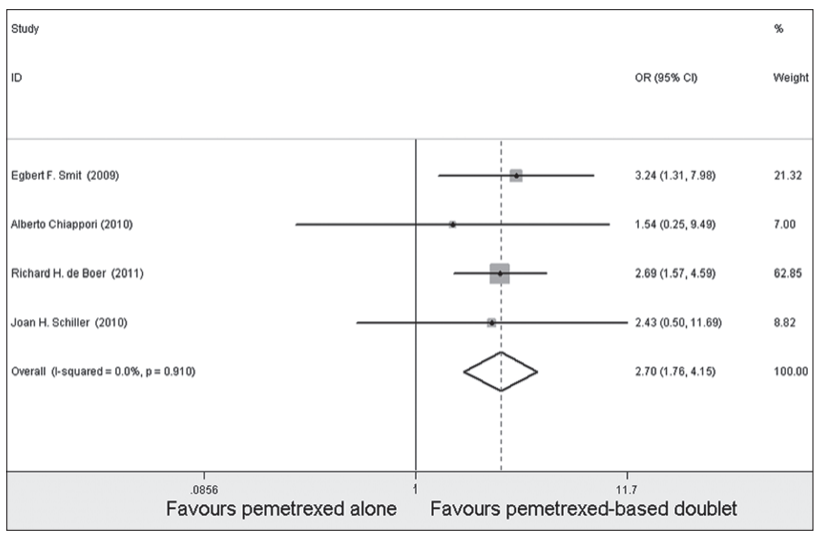

Fig. 4. Forest plot from meta-analysis of overall response rate associated with pemetrexed-based doublet compared with pemetrexed alone.

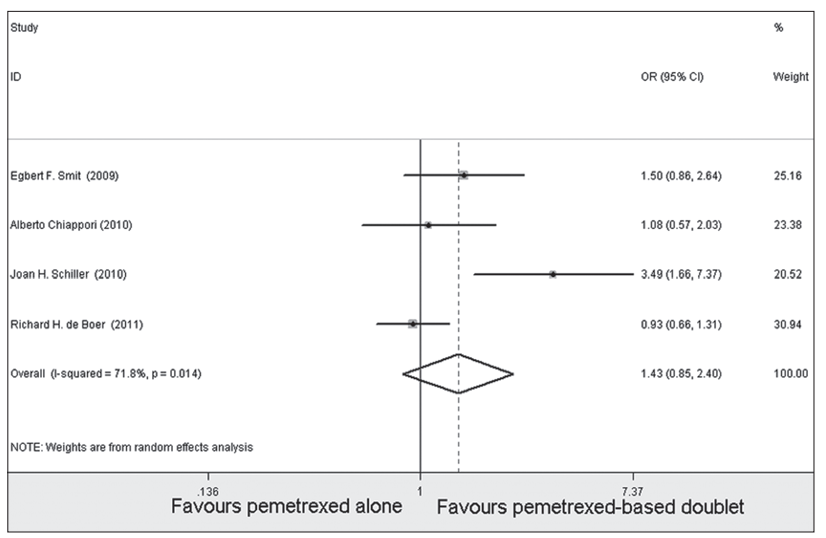

Fig. 5. Forest plot from meta-analysis of 1-year survival rate associated with pemetrexed-based doublet compared with pemetrexed alone.

in these four trails for data analysis. Pemetrexed-based doublet chemotherapy regimens in the four trails were as follows: pemetrexed and carboplatin (11); pemetrexed and enzastaurin (14); pemetrexed and vandetanib (15); pemetrexed and matuzumab (12). In two RCT trails $(14,15)$, regimens of pemetrexed alone groups were pemetrexed plus placebo, while the other two trails $(11,12)$ did not have placebo. All four trails compared pemetrexed alone with pemetrexed-based doublet regimens. The characteristics of these studies are listed in Table 1. Jadad scores were 3, 4, 5, 3 points, respectively.

\section{Overall survival}

The pooled HR for OS showed that there was no significant difference in overall survival between pemetrexed-based doublets with pemetrexed alone arm (HR $=0.88,95 \%$ CI: $0.74-1.04$, $\mathrm{p}=0.132$ ) (Fig. 2). Test for heterogeneity did not show significant difference $(\mathrm{p}=0.622$ ), and fixed-effort model was used to calculate pooled HR for OS.

\section{Progression-free survival}

The pooled HR for PFS did not show significant difference in progression-free survival between pemetrexed-based doublets regi- 


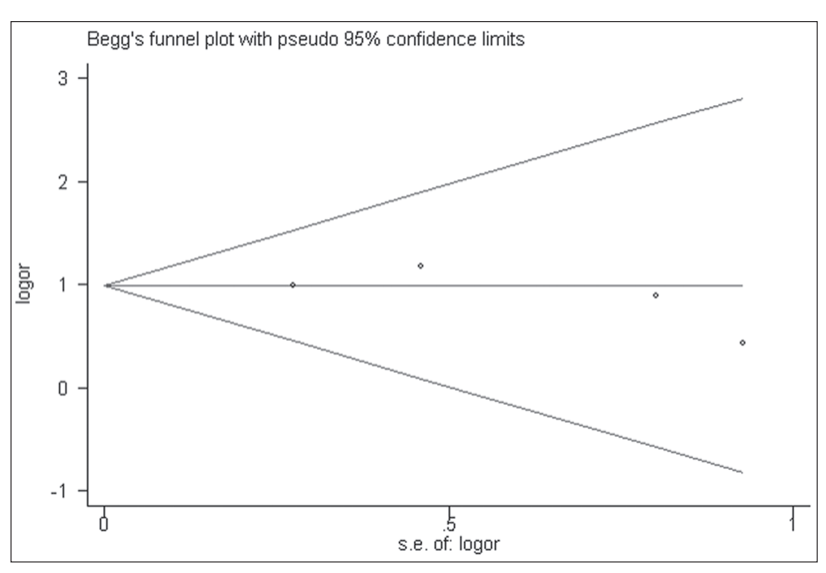

Fig. 6. Funnel plot of publication bias in the meta-analysis.

mens group with single-agent pemetrexed group $(\mathrm{HR}=0.91,95 \%$ CI: $0.73-1.15, p=0.443$ ) (Fig. 3). Test for heterogeneity showed that there was significant difference $(\mathrm{p}=0.030)$, and random-effort model was used to calculate pooled HR for PFS.

\section{Overall response rate}

The pooled OR for ORR showed that there was significant difference in overall response rate between pemetrexed-based doublets and pemetrexed alone group (pemetrexed-based doublet group significantly improved overall response rate) $(\mathrm{OR}=2.70$, 95\% CI: 1.76-4.15, p=0.000) (Fig. 4). Test for heterogeneity did not show significant difference $(\mathrm{p}=0.910)$, while fixed-effort model was used to calculate pooled OR for ORR.

\section{1-year survival rate}

The pooled OR for 1-year survival rate showed that there was no significant difference in 1-year survival rate between pemetrexed-based doublets and pemetrexed alone group $(\mathrm{OR}=1.43,95 \%$ CI: $0.85-2.40, p=0.178$ ) (Fig. 5). Test for heterogeneity showed that there was significant difference $(\mathrm{p}=0.014)$, while random-effort model was used to calculate pooled OR for 1-year survival rate.

\section{Safety}

The pooled OR for grade 3-4 leucopenia, neutropenia and thrombocytopenia showed that there were statistically significantly higher frequencies in pemetrexed-based doublet group than in single-agent pemetrexed arm $(\mathrm{OR}=2.86,95 \% \mathrm{CI}$ : $1.32-6.20$, $\mathrm{p}=0.008 ; \mathrm{OR}=2.69,95 \% \mathrm{CI}: 1.55-4.68, \mathrm{p}=0.000 ; \mathrm{OR}=6.92,95 \%$ CI: $2.51-19.07, \mathrm{p}=0.000$; respectively) (Tab. 2). The pooled OR for grade 3-4 anemia and fatigue showed that there were no statis- tically significant differences in frequencies of anemia and fatigue between the two groups $(\mathrm{OR}=0.62,95 \% \mathrm{CI}$ : $0.33-1.18, \mathrm{p}=0.144$; $\mathrm{OR}=1.15,95 \%$ CI: $0.73-1.79, \mathrm{p}=0.550$; respectively).

\section{Publication bias}

There was on publication bias according to funnel plot (Begg's test, $\mathrm{p}=0.308$; Egger test, $\mathrm{p}=0.468$ ) (Fig. 6).

\section{Discussion}

Progression is nearly inevitable in advanced NSCLC patients, and approximately thirty to forty percent of those patients received second-line chemotherapy after standard first-line treatment $(16,17)$. Due to similar clinical efficacy and significantly fewer side effects, single-agent pemetrexed was recommended as the second-line treatment for recurrent NSCLC patients. Pemetrexed is a novel chemotherapeutic drug that could inhibit the thymidylate synthase, dihydrofolate reductase, and glycinamide ribonucleotide formyl transferase to prevent tumor cell growth (8). However, the response rate in second-line treatment of NSCLC is low (less than $10 \%$ with chemotherapy) and survival time is limited $(18,19)$.

One of the possible logical strategies to improve the efficacy of second-line treatment is combination chemotherapy. In 2007 Weiss et al (20) reported that in their retrospective single institution analysis with pemetrexed and bevacizumab failed after firstline therapy compared with pemetrexed alone in patients with advanced NSCLC. The results showed that there were no significant differences in overall survival $(\mathrm{p}=0.798)$ and TTP (time to progression $)(\mathrm{p}=0.6125)$ between pemetrexed and bevacizumab group with pemetrexed alone arm, and there were no grade 3-5 bleeding events reported. However, the study had a small sample size of 25 patients and was not RCTs but retrospective study. For that reason, this study had insufficient power to detect whether pemetrexed-based doublet was superior to single-agent pemetrexed as second-line treatment for NSCLC. Therefore, a meta-analysis of pemetrexed or pemetrexed-based doublet therapy should be performed in order to contribute to NSCLC treatment fields. The purpose of our meta-analysis was to assess whether patients could gain more benefit from pemetrexed-based doublet compared with single-agent in second-line treatment of NSCLC.

Our results showed that there were no significant differences between pemetrexed-based doublet and single-agent pemetrexed in OS (HR $=0.88,95 \% \mathrm{CI}: 0.74-1.04, \mathrm{p}=0.132)$, PFS $(\mathrm{HR}=0.91,95 \%$ CI: $0.73-1.15, \mathrm{p}=0.443)$ and 1 -year survival rate $(\mathrm{OR}=1.23,95 \%$ CI: $0.96-1.58, \mathrm{p}=0.100)$. This indicates that pemetrexed-based

Tab. 2. Summary of Grade 3-4 toxicity meta-analyses of pemetrexed-based doublet compared with pemetrexed alone for advanced NSCLC.

\begin{tabular}{|c|c|c|c|c|c|c|c|}
\hline Toxicity & Trials & $\begin{array}{c}\text { pemetrexed-based } \\
\text { doublet chemotherapy }\end{array}$ & $\begin{array}{l}\text { single-agent } \\
\text { pemetrexed }\end{array}$ & $\begin{array}{c}\text { P-value for } \\
\text { homogeneity }\end{array}$ & OR & $95 \% \mathrm{CI}$ & P-value \\
\hline Grade 3-4 anemia & 3 & $16 / 458$ & $26 / 474$ & 0.016 & 0.62 & $0.33-1.18$ & 0.144 \\
\hline Grade 3-4 neutropenia & 3 & $55 / 296$ & $19 / 251$ & 0.514 & 2.69 & $1.55-4.68$ & 0.000 \\
\hline Grade 3-4 thrombocytopenia & 3 & $29 / 296$ & $4 / 251$ & 0.981 & 6.92 & $2.51-19.07$ & 0.000 \\
\hline Grade $3-4$ fatigue & 4 & $46 / 556$ & $40 / 524$ & 0.682 & 1.15 & $0.73-1.79$ & 0.550 \\
\hline Grade 3-4 leucopenia & 3 & $27 / 296$ & $9 / 251$ & 0.530 & 2.86 & $1.32-6.20$ & 0.008 \\
\hline
\end{tabular}


doublet therapy was not superior to single-agent pemetrexed, while patients who received pemetrexed-based doublet had a markedly increased ORR $(\mathrm{OR}=2.70,95 \% \mathrm{CI}: 1.76-4.15, \mathrm{p}=0.000)$ compared with those receiving single-agent pemetrexed.

More adverse events were detected in pemetrexed-based doublet arm. The meta-analysis showed that there were more frequent adverse events including leucopenia $(\mathrm{OR}=2.86,95 \%$ CI: $1.32-6.20, p=0.008)$, neutropenia $(\mathrm{OR}=2.69,95 \%$ CI: $1.55-$ $4.68, \mathrm{p}=0.000)$ and thrombocytopenia $(\mathrm{OR}=6.92,95 \% \mathrm{CI}: 2.51-$ $19.07, p=0.000$ ) in pemetrexed-based doublet group, while grade 3-4 anemia and fatigue in pemetrexed-based doublet arm were equivalent in frequencies compared with those in single-agent therapy.

The interpretation of results of our meta-analysis should be cautious because the study included only four RCTs, they contained small number of patients, and the 1-year survival rate in all four eligible RCTs were deciphered from the survival curve due to lack of this information in above four literatures. Although there were limitations in our analysis, it may assist us, oncologists, in proper cognition of efficacy and toxicity of pemetrexed-based doublet chemotherapy in advanced NSCLC. We reviewed literature in English and found that there was no meta-analysis similar to ours. Thus, our study was the first meta-analysis to compare tpemetrexed-based doublet with single-agent pemetrexed in second-line therapy of NSCLC.

In conclusion, despite the fact that our meta-analysis showed a markedly increased ORR in pemetrexed-based doublet arm, pemetrexed combination chemotherapy was not recommended as the second-line chemotherapy for patients of NSCLC due to lack of survival benefit and increased toxicity.

\section{References}

1. Hu Z, Chen X, Zhao Y, Tian T, Jin G et al. Serum microRNA signatures identified in a genome-wide serum microRNA expression profiling predict survival of non-small-cell lung cancer. J Clin Oncol 2010; 28: 1721-1726.

2. Gao G, Chu H, Zhao L, Gui T, Xu Q et al. A meta-analysis of paclitaxel-based chemotherapies administered once every week compared with once every 3 weeks first-line treatment of advanced non-small-cell lung cancer. Lung Cancer 2012; 76: 380-386.

3. Novello S, Le Chevalier T. Chemotherapy for non-small-cell lung cancer. Part 1: Early-stage disease. Oncology (Williston Park) 2003; 17 : $357-364$.

4. Gridelli C, Rossi A, Morgillo F, Bareschino MA, Maione P et al. A randomized phase II study of pemetrexed or RAD001 as second-line treatment of advanced non-small-cell lung cancer in elderly patients: treatment rationale and protocol dynamics. Clin Lung Cancer 2007; 8: 568-571.

5. Noble J, Ellis PM, Mackay JA, Evans WK. Second-line or subsequent systemic therapy for recurrent or progressive non-small cell lung cancer: a systematic review and practice guideline. J Thorac Oncol 2006; 1: $1042-1058$.

6. Qi WX, Shen Z, Yao Y. Meta-analysis of docetaxel-based doublet versus docetaxel alone as second-line treatment for advanced non-small-cell lung cancer. Cancer Chemother Pharmacol 2012; 69: 99-106.
7. Shepherd FA, Dancey J, Ramlau R, Mattson K, Gralla R et al. A prospective randomized trial of docetaxel versus best supportive care in patients with non-small-cell lung cancer previously treated with platinumbased chemotherapy. J Clin Oncol 2000; 18: 2095-2103.

8. Hanna N, Shepherd FA, Fossella FV, Pereira JR, De Marinis F et al. Randomized phase III trial of pemetrexed versus docetaxel in patients with non small cell lung cancer previously treated with chemotherapy. J Clin Oncol 2004; 22: 1589-1597.

9. Barlési F, Jacot W, Astoul P, Pujol JL. Second-line treatment for advanced non-small cell lung cancer: a systematic review. Lung Cancer 2006; 51: 159-172.

10. Schuette W, Tesch H, Büttner H, Krause T, Soldatenkova V et al. Second-line treatment of stage III/IV non-small-cell lung cancer (NSCLC) with pemetrexed in routine clinical practice: evaluation of performance status and health-related quality of life. BMC Cancer 2012; 12: 14.

11. Smit EF, Burgers SA, Biesma B, Smit HJ, Eppinga $P$ et al. Randomized phase II and pharmacogenetic study of pemetrexed compared with pemetrexed plus carboplatin in pretreated patients with advanced non-small-cell lung cancer. J Clin Oncol 2009; 27: 2038-2045.

12. Schiller JH, von Pawel J, Schütt P, Ansari RH, Thomas M et al. Pemetrexed with or without matuzumab as second-line treatment for patients with stage IIIB/IV non-small cell lung cancer. J Thorac Oncol 2010; 5: 1977-1985.

13. Moher D, Pham B, Jones A, Cook DJ, Jadad AR et al. Does quality of reports of randomised trials affect estimates of intervention efficacy reported in meta-analyses? Lancet 1998; 352: 609-613.

14. Chiappori A, Bepler G, Barlesi F, Soria JC, Reck M et al. Phase II, double-blinded, randomized study of enzastaurin plus pemetrexed as second-line therapy in patients with advanced non-small cell lung cancer. J Thorac Oncol 2010; 5: 369-375.

15. de Boer RH, Arrieta Ó, Yang CH, Gottfried M, Chan V et al. Vandetanib plus pemetrexed for the second-line treatment of advanced nonsmall-cell lung cancer: a randomized, double-blind phase III trial. Clin Oncol 2011; 29: 1067-1074.

16. Scagliotti GV, De Marinis F, Rinaldi M, Crinò L, Gridelli C et al. Phase III randomized trial comparing three platinum-based doublets in advanced non-small cell lung cancer. J Clin Oncol 2002; 20: 4285-4291.

17. Gatzemeier U, von Pawel J, Gottfried M, ten Velde GP, Mattson K et al. Phase III comparative study of high-dose cisplatin versus a combination of paclitaxel and cisplatin in patients with advanced non-small-cell lung cancer. J Clin Oncol 2000; 18: 3390-3399.

18. de Marinis F, Pereira JR, Fossella F, Perry MC, Reck M et al. Lung Cancer Symptom Scale outcomes in relation to standard efficacy measures: an analysis of the phase III study of pemetrexed versus docetaxel in advanced non-small cell lung cancer. J Thorac Oncol 2008; 3: 30-36.

19. Tassinari D, Scarpi E, Sartori S, Tamburini E, Santelmo C et al. Second-line treatments in non-small cell lung cancer. A systematic review of literature and metaanalysis of randomized clinical trials. Chest 2009; 135: 1596-1609.

20. Weiss GJ, Zeng C, Kelly K, Tran ZV, Bunn PAJr. Single-institution experience with pemetrexed and bevacizumab as salvage therapy in advanced non-small-cell lung cancer. Clin Lung Cancer 2007; 8: 335-338.

Received October 6, 2012. Accepted October 27, 2013. 